\title{
Distribution of Parametric Conductance Derivatives of a Quantum Dot
}

\author{
P W Brouwer, ${ }^{1} \mathrm{~S}$ A van Langen, ${ }^{1} \mathrm{~K}$ M Frahm, ${ }^{1} *$ M Buttker, ${ }^{2}$ and C W J Beenakker ${ }^{1}$ \\ ${ }^{1}$ Instutuut Lorentz Leiden University PO Box 95062300 RA Leiden The Netherlands \\ ${ }^{2}$ Departement de Physique Theorique Untversite de Geneve 1211 Geneve 4 Switzerland
}

(Received 24 February 1997)

\begin{abstract}
The conductance $G$ of a quantum dot with single-mode ballistic point contacts depends sensitively on external parameters $X$ such as gate voltage and magnetıc field We calculate the joint distribution of $G$ and $d G / d X$ by relating it to the distribution of the Wigner Smith time delay matrix of a chaotic system The distribution of $d G / d X$ has a singularity at zero and algebraic tails While $G$ and $d G / d X$ are correlated, the ratio of $d G / d X$ and $\sqrt{G(1-G)}$ is independent of $G$ Coulomb interactions change the distribution of $d G / d X$ by inducing a transition from the grand canonical to the canonical ensemble All these piedictions can be tested in semiconductor microstiuctures or microwave cavities [S0031 9007(97)03744 7]

PACS numbers 7323 -b $0545+b 4225$ Bs $8530 \mathrm{Vw}$
\end{abstract}

Parametric fluctuations in quantum systems with a chaotic classical dynamics are of fundamental importance for the characterization of mesoscopic systems The fluctuating dependence of an energy level $E_{l}(X)$ on an external parameter $X$, such as the magnetic field, has received considerable attention [1] A key role is played by the "level velocity" $d E_{J} / d X$, describing the response to a small perturbatıon [2-4] In open systems, the role of the level velocity is played by the "conductance velocity" $d G / d X$ Remarkably little is known about its distribution

The interest in this problem was stımulated by experıments on semiconductor microstructures known as quantum dots, in which the election motion is ballistic and chaotic [5] A typical quantum dot is confined by gate electrodes, and connected to two election reservours by bal listic point contacts, thiough which only a few modes can propagate at the Fermı level The parametı ic dependence of the conductance has been measured by several groups [6-8] In the single-mode limit, parametric fluctuations are of the same order as the average, so that one needs the complete distribution of $G$ and $d G / d X$ to character ize the system Knowing the average and variance is not sufficient Analytical results are avallable for point con tacts with a large number of modes [9-15] In this paper, we present the complete distribution in the opposite limit of two single-mode point contacts and show that it differs strikıngly from the multımode case considered previously

The main differences which we have found are the following We consider the joint distribution of the conductance $G$ and the derivatives $\partial G / \partial V, \partial G / \partial X$ with respect to the gate voltage $V$ and an external parameter $X$ (typically the magnetic field) If the point contacts contain a large number of modes, $P(G, \partial G / \partial V, \partial G / \partial X)$ factorizes into three independent Gaussian distributions [9-12] In the single-mode case, in contrast, we find that this distribution does not factorize and decays algebraically rather than exponentially By integratıng out $G$ and one of the two derivatıves, we obtain the conductance velocity distributions $P(\partial G / \partial V)$ and $P(\partial G / \partial X)$ plotted in Fig 1 Both distıbutions have a singularity at zero velocity, and alge braic tals A rematkable prediction of our theory is that the correlations between $G$, on the one hand, and $\partial G / \partial V$ and $\partial G / \partial X$, on the other hand, can be transformed away by the change of variables $G=\left(2 e^{2} / h\right) \sin ^{2} \theta$, where $\theta$ is the polar coordinate introduced in Ref [13] The derivatives $\partial \theta / \partial V$ and $\partial \theta / \partial X$ are statistically independent of $\theta$ There exists no change of variables that transforms away the correlations between $\partial G / \partial V$ and $\partial G / \partial X$

Another new feature of the single mode case concerns the effect of Coulomb inter actions [16,17] In the simplest model, the strength of the Coulomb repulsion is measured by the ratio of the charging energy $e^{2} / C$ (with $C$ the capacitance of the quantum dot) and the mean level spacing $\Delta$ In the regime $e^{2} / C \gg \Delta$, where most experiments are done, Coulomb interactions suppress fluctuations of the charge $Q$ on the quantum dot as a function of $V$ or $X$, at the expense of fluctuations in the electrical potential $U$ Since the Ferm level $\mu$ in the quantum dot is pinned by the reservoirs, the kinetic energy $E=\mu-U$ at the Fermi level fluctuates as well Fluctuations of $E$ cannot be ignored, because the conductance is determined by $E$, and not by $\mu$ An ensemble of quantum dots with fixed $Q$ and fluctuatıng $E$ behaves effectively as a canonical ensemble - rather than a grand-canonical ensemble In the opposite regime $e^{2} / C \ll \Delta$, the energy $E$ does not fluctuate on the scale of the level spacing The ensemble is now truly grand-canonical Fluctuations of $E$ on the scale of $\Delta$ can be neglected in the multımode case, so that the distinction between canonical and grand-canonical averages is irrelevant In the single-mode case the distinction becomes important We will see that the distribution of the conductance velocities is different in the two ensembles (The distribution of the conductance itself is the same ) The difference between grand-canonical and canonical av erages has been studied extensively in connection with the problem of the persistent current [18-20], which is a thermodynamic property Here we find a difference in the case of a transport property, which is more unusual $[21,22]$ 

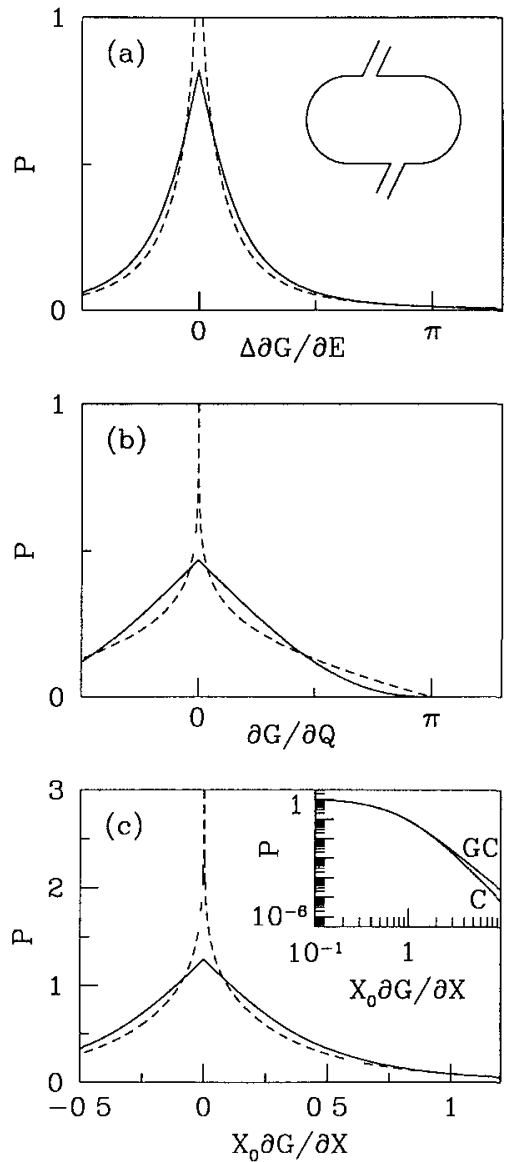

FIG 1 Distributions of conductance velocities in a chaotic cavity with two single-mode point contacts [inset in (a)], computed from Eq (10) Dashed curves are for $\beta=1$ (timereversal symmetry), solid curves for $\beta=2$ (no time-reveısal symmetry) (The case $\beta=4$, which is similar to $\beta=2$, is omitted for clanty) The distribution of $\Delta \partial G / \partial E$ (grand canonical ensemble) is shown in (a) and the distisbution of $\partial G / \partial Q$ (canonical ensemble) is shown in (b) (The conductance $G$ is measured in units of $2 e^{2} / h$, the charge $Q$ in units of $e$ ) In (c) the distribution of $X_{0} \partial G / \partial X$ is shown for the grandcanonical ensemble (the canonical case being nearly identical on a linear scale) The inset compares the canonical (C) and grand-canonical (GC) results for $\beta=2$ on a logarithmıc scale

To derive these results, we combine a scattering formalism with random-matrix theory [23] The $2 \times 2$ scattering matrix $S$ determines the conductance

$$
G=\left|S_{12}\right|^{2},
$$

and the (unscreened) compressibilities [17]

$$
\frac{\partial Q}{\partial E}=\frac{1}{2 \pi l} \operatorname{tr} S^{\dagger} \frac{\partial S}{\partial E}, \quad \frac{\partial Q}{\partial X}=\frac{1}{2 \pi \imath} \operatorname{tr} S^{\dagger} \frac{\partial S}{\partial X}
$$

(We measure $G$ in units of $2 e^{2} / h$ and $Q$ in units of $e$ ) Grand-canonical averages \langle\rangle$_{G C}$ and canonical averages \langle\rangle$_{C}$ are related by

$$
\langle\rangle_{C}=\Delta\langle\quad \times d Q / d E\rangle_{G C}
$$

The factor $d Q / d E$ is the Jacobian to go from an average over $Q$ in the canonical ensemble to an average over $E$ in the grand-canonical ensemble Conductance velocities in the two ensembles are related by

$$
\left.\frac{\partial G}{\partial X}\right|_{Q}=\left.\frac{\partial G}{\partial X}\right|_{E}-\frac{\partial G}{\partial E} \frac{\partial Q}{\partial X}\left(\frac{\partial Q}{\partial E}\right)^{-1},
$$

where $\left.\right|_{Q}$ and $\left.\right|_{E}$ indicate, respectively, derivatives at constant $Q$ (canonical) and constant $E$ (grand-canonical) Derivatives $\partial G / \partial V$ with respect to the gate voltage are proportional to $\partial G / \partial Q$ in the canonical ensemble and to $\partial G / \partial E$ in the grand-canonical ensemble (The proportionality coefficients contain elements of the capacitance matrix of the quantum dot plus gates ) The two derivatives are related by

$$
\frac{\partial G}{\partial Q}=\frac{\partial G}{\partial E}\left(\frac{\partial Q}{\partial E}\right)^{-1}
$$

The problem that we face is the calculation of the joint distribution of $S, \partial S / \partial E$, and $\partial S / \partial X$ In view of the relations (3)-(5) it is sufficient to consider the grandcanonical ensemble This problem is closely related to the old problem [24] of the distribution of the WignerSmith delay tımes $\tau_{1}, \quad, \tau_{N}$, which are the eigenvalues of the $N \times N$ matrix $-{ }_{l} S^{\dagger} \partial S / \partial E$ (The eigenvalues are real positive numbers ) Interest in this problem has been revived in connection with chaotic scattering [25-28] The rates $\gamma_{n}=1 / \tau_{n}$ are distributed accordıng to [28]

$$
P\left(\left\{\gamma_{n}\right\}\right) \propto \prod_{t<j}\left|\gamma_{l}-\gamma_{j}\right|^{\beta} \prod_{k} \gamma_{k}^{\beta N / 2} e^{-\pi \beta \gamma_{k} / \Delta}
$$

This distribution is known in random-matrix theory as the Laguerre ensemble, because the correlation functions can be wiltten as se11es over (generalized) Laguerre polynomı als [29] For $N=1$ we 1ecover the 1esult of Refs [25] and [27] In our case $N=2$

To compute the conductance velocities it is not sufficient to know the delay times $\tau_{n}$, but we also need to know the distribution of the eigenvectors of the time-delay mat11x $-{ }_{t} S^{\dagger} \partial S / \partial E$ Furthermore, we need the distribution of $-{ }_{l} S^{\dagger} \partial S / \partial X$ The general result containing this infotmation is [28]

$$
\begin{aligned}
P\left(S, \tau_{L}, \tau_{X}\right) \propto & \exp \left[-\beta \operatorname{tr}\left(\frac{\pi}{\Delta} \tau_{E}^{-1}+\frac{\pi^{2} X_{0}^{2}}{4 \Delta^{2}}\left(\tau_{E}^{-1} \tau_{X}\right)^{2}\right)\right] \\
& \times\left(\operatorname{det} \tau_{E}\right)^{-2 \beta N+3(\beta-2) / 2},
\end{aligned}
$$

$\tau_{E}=-{ }_{l} S^{-1 / 2} \frac{\partial S}{\partial E} S^{1 / 2}, \quad \tau_{X}=-{ }_{l} S^{-1 / 2} \frac{\partial S}{\partial X} S^{-1 / 2}$

The matrix $\tau_{L}$ has the same eigenvalues as the time-delay matrix, but it is more convenient because it is uncolrelated with $S$, while the time-delay matrix is not By integrating out $\tau_{E}$ and $\tau_{X}$ from Eq (7), we obtain a un1form distribution for $S$, as expected for a chaotic cavity [30] The resulting distribution of the conductance [31], 
$P(G) \propto G^{-1+\beta / 2}$, is the same in the canonical and grandcanonical ensembles, because $S$ and $d Q / d E$ are uncorrelated [cf Eq (3)] By integratıng out $S, \tau_{X}$, and the eigenvectors of $\tau_{E}$, we obtain the distribution (6) of the delay times The distribution of $\tau_{X}$ at fixed $\tau_{E}$ is a Gaussian The scale of this Gaussian is set by the parameter $X_{0}$, which has no universal value [32]

We are now ready to compute the distribution of the conductance velocities Derivatives with respect to $E$ and $Q$ are related to the delay times by

$$
\begin{aligned}
& \frac{\partial G}{\partial E}=c\left(\tau_{1}-\tau_{2}\right) \sqrt{G(1-G),} \\
& \frac{\partial G}{\partial Q}=2 \pi c \frac{\tau_{1}-\tau_{2}}{\tau_{1}+\tau_{2}} \sqrt{G(1-G)},
\end{aligned}
$$

where $c \in[-1,1]$ is a number that depends on the phases of the matrix elements of $S$ and on the ergenvectors of $\tau_{E}$
Its distribution $P(c) \propto\left(1-c^{2}\right)^{-1+\beta / 2}$ is independent of $\tau_{1}, \tau_{2}$, and $G$ The derivatıve $\partial G / \partial X$ has a Gaussian distribution at a given value of $S$ and $\tau_{E}$, with zero mean and with variance

$$
\begin{aligned}
& \left\langle\left(\left.\frac{\partial G}{\partial X}\right|_{E}\right)^{2}\right\rangle=\alpha\left[G(1-G) \tau_{1} \tau_{2}+\frac{1}{2}\left(\frac{\partial G}{\partial E}\right)^{2}\right], \\
& \left\langle\left(\left.\frac{\partial G}{\partial X}\right|_{Q}\right)^{2}\right\rangle=\alpha \tau_{1} \tau_{2}\left[G(1-G)-\frac{1}{4 \pi^{2}}\left(\frac{\partial G}{\partial Q}\right)^{2}\right],
\end{aligned}
$$

where we have abbreviated $\alpha=4 \Delta^{2} / \pi^{2} X_{0}^{2} \beta \quad$ Because the variance of $\partial G / \partial X$ depends on $\partial G / \partial E$ or $\partial G / \partial Q$, these conductance velocities are correlated

From the distribution (6) of $\tau_{1}, \tau_{2}$, and the independent distributions of $G$ and $c$, we calculate the joint distribution of $G$ and its (dimensionless) derivatives $G_{X}=X_{0} \partial G / \partial X$, $G_{E}=(\Delta / 2 \pi) \partial G / \partial E$, and $G_{Q}=(1 / 2 \pi) \partial G / \partial Q$ The result in the grand-canonical and canonical ensembles is

$$
\begin{aligned}
P_{G C}\left(G, G_{E}, G_{X}\right) & =\frac{1}{Z} \int_{0}^{\infty} d x \int_{G_{E}^{2} /[G(1-G)]}^{\infty} d y \frac{\left[y G-G_{E}^{2} /(1-G)\right]^{-1+\beta / 2} x^{-2-2 \beta}}{\sqrt{\pi(x+y) G(1-G) f(x)}} \exp \left[-\frac{2 \beta}{x} \sqrt{x+y}-\frac{G_{X}^{2}}{f(x)}\right], \\
P_{C}\left(G, G_{Q}, G_{X}\right) & =\frac{2}{Z} \int_{0}^{\infty} d x \int_{G_{Q}^{2} /[G(1-G)]}^{1} d y \frac{\left[y G-G_{Q}^{2} /(1-G)\right]^{-1+\beta / 2} x^{3 \beta}}{(1-y)^{(\beta+3) / 2} \sqrt{\pi G(1-G) g(x)}} \exp \left[-\frac{2 \beta x}{\sqrt{1-y}}-\frac{G_{X}^{2}}{g(x)}\right], \\
f(x) & =8 \beta^{-1}\left[x G(1-G)+2 G_{E}^{2}\right], \quad g(x)=8\left(x^{2} \beta\right)^{-1}\left[G(1-G)-G_{Q}^{2}\right], \\
Z & =3 \beta^{-3 \beta-1} \Gamma(\beta / 2) \Gamma(\beta) \Gamma(3 \beta / 2)
\end{aligned}
$$

By integrating out $G$ and one of the two denivatives from Eq (10), we obtain the conductance velocity distributions of Fig 1 (The case $\beta=4$ is close to $\beta=2$ and is omitted from the plot for clarity ) The disti1butions have a singulanity at zeıo derivatıve A cusp for $\beta=2$ and 4 , and a logatithmic divergence for $\beta=1$ The talls of the distributions of $\partial G / \partial X$ are algebraic in both ensembles, but with a different exponent,

$$
\begin{aligned}
& P_{G C}(\partial G / \partial X) \propto(\partial G / \partial X){ }^{\beta}{ }^{2}, \\
& P_{C}(\partial G / \partial X) \propto(\partial G / \partial X)^{2 \beta} 1
\end{aligned}
$$

The distribution of $\partial G / \partial E$ (grand-canonical ensemble) also has an algebraic tall $\left[\propto(\partial G / \partial E)^{-\beta-2}\right]$, while the distribution of $\partial G / \partial Q$ (canonical ensemble) is identically zero for $|\partial G / \partial Q| \geq \pi$ In both ensembles, the second moment of the conductance velocities is finite for $\beta=2$ and 4 , but infinite for $\beta=1$ [33]

In conclusion, we have calculated the joint distribution of the conductance $G$ and its parametric derivatives for a chaotic cavity, coupled to electron reservorrs by two single-mode ballistic point contacts The distribution is fundamentally different from the multimode case, being highly non-Gaussian and with correlated deiıvatives (Correlations between $G$ and the parametric derivatives can be transformed away by a change of variables ) We account tor Coulomb interactions by using a canonical ensemble instead of a grand-canonical ensemble Our results for the canonical ensemble are relevant for the analysis of recent experiments on chaotic quantum dots, where the conductance $G$ is measured as a function of both the magnetic field and the shape of the quantum dot [8] The grand canonical results are relevant for experiments on microwave cavities $[34,35]$ Together with the theory provided here, such experiments can yield information on the distribution of delay times in chaotic scattering that cannot be obtained by other means

This problem was suggested to us by $\mathrm{C} M$ Marcus We acknowledge support by the Dutch Science Founda tion NWO/FOM and by the European Community (program for the Trainıng and Mobılity of Researchers)

+Pıesent address Laboratorre de Physique Quantıque, UMR 5626 du CNRS, Univeisite Paul Sabatier, 31062 Toulouse Cedex 4, Fiance

[1] B L Altshuler and B D Simons, in Mesoscoplc Quantum Physacs edited by E Akkermans, G Montambaux, J-L Pichard, and J Zinn Justın (North-Holland, Amsterdam, 1995) 
[2] B D Simons and B L Altshuler, Phys Rev B 48, 5422 (1993)

[3] Y V Fyodorov, Phys Rev Lett 73, 2688 (1994), Y V Fyodorov and A D Mirlin, Phys Rev B 51, 13403 (1995)

[4] N Tanıuchı, A Hashımoto, B D Simons, and B L Altshuler, Europhys Lett 27, 335 (1994)

[5] R M Westervelt, in Nano-Science and Technology, edited by G Timp (American Institute of Physics, New York, 1996)

[6] C M Marcus, A J Rimberg, R M Westervelt, P F Hopkins, and A C Gossard, Phys Rev Lett 69, 506 (1992)

[7] A M Chang, $\mathrm{H} \mathrm{U}$ Baranger, L N Pfeiffer, and $\mathrm{K} \mathrm{W}$ West, Phys Rev Lett 73, 2111 (1994)

[8] I H Chan, R M Clarke, C M Marcus, K Campman, and A C Gossard, Phys Rev Lett 74, 3876 (1995)

[9] R A Jalabert, H U Baranger, and A D Stone, Phys Rev Lett 65, 2442 (1990)

[10] Z Pluhăr, H A Weidenmuller, J A Zuk, and $\mathrm{C} \mathrm{H}$ Lewenkopf, Phys Rev Lett 73, 2115 (1994)

[11] K B Etetov, Phys Rev Lett 74, 2299 (1995)

[12] K Frahm, Europhys Lett 30, 457 (1995)

[13] K Frahm and J-L P1chard, J Phys I (France) 5, 877 (1995)

[14] J Rau, Phys Rev B 51, 7734 (1995)

[15] A M S Macêdo, Phys Rev B 53, 8411 (1996)

[16] M Buttıker, J Phys Condens Matter 5, 9361 (1993)

[17] M Buttiker and T Christen, in Quantum Transport in Semiconductor Submicron Structures, edited by B Kramer, NATO ASI Ser E, Vol 326 (Kluwer, Dordrecht, 1996)

[18] H -F Cheung, Y Gefen, E K Riedel, and W-H Shıh, Phys Rev B 37, 6050 (1988)

[19] H Bouchiat and G Montambaux, J Phys (Parı) 50, 2695 (1989)

[20] B L Altshuler, Y Gefen, and Y Imry, Phys Rev Lett 66, 88 (1991)

[21] A Kamenev and Y Gefen, Phys Rev B 49, 14474 (1994)
[22] The difference between canonical and grand-canonical averages which we find is related, but not identical, to the effects of the Coulomb blockade predicted by I L Aleiner and L I Glazman, Report No cond-mat/9612138

[23] C W J Beenakker, Report No cond-mat/9612179

[24] E P Wigner, Phys Rev 98, 145 (1955), F T Smith, Phys Rev 118, 349 (1960)

[25] Y V Fyodorov and H -J Sommers, Phys Rev Lett 76, 4709 (1996), J Math Phys 38, 1918 (1997)

[26] P Šeba, K Zyczkowskı, and J Zakrewskı, Phys Rev E 54, 2438 (1996)

[27] V A Gopar, P A Mello, and M Buttıker, Phys Rev Lett 77, 3005 (1996)

[28] P W Brouwer, K M Frahm, and C W J Beenakker, Phys Rev Lett 78, 4737 (1997)

[29] T Nagao and K Slevin, J Math Phys 34, 2075 (1993), T Nagao and P J Forrester, Nucl Phys B435, 401 (1995)

[30] R Blumel and U Smllansky, Phys Rev Lett 60, 477 (1988)

[31] H U Baranger and P A Mello, Phys Rev Lett 73, 142 (1994), R A Jalabert, J -L Pichaid, and C W J Beenakker, Europhys Lett 27, 255 (1994)

[32] If $X$ represents the magnetic flux through the quantum dot, then $X_{0} \simeq(h / e)\left(\tau_{\text {crgodic }} / \tau_{\mathrm{H}}\right)^{1 / 2}$, where $\tau_{\mathrm{H}}$ is the Heisenberg time of the quantum dot and $\tau_{\text {ctgodic }} \ll \tau_{H}$ is the time scale for ergodic exploration of the avalable phase space

[33] The algebraic tails of the distributions of $\partial G / \partial E$ and $\partial G / \partial X$, and hence the divergencies of the second moments, are cut off by thermal smearing or inelastrc scattering at $\left(X_{0} / \Delta\right) \partial G / \partial X \sim \partial G / \partial E \sim \min \left(\tau_{\phi}, 1 / k T\right)$, where $\tau_{\phi}$ is the dephasing time and $T$ the temperature At the same time, the cusp neal zero denvative is rounded off The bulk of the distribution remains unchanged as long as $\max \left(1 / \tau_{\phi}, k T\right) \leq \Delta$

[34] J Stein, H -J Stockmann, and U Stoffregen, Phys Rev Lett 75, 53 (1995)

[35] A Kudrollı, V Kidambi, and S Sildhar, Phys Rev Lett 75, 822 (1995) 